\title{
La Tecnología Educativa en la Pedagogía del siglo XXI: una visión en 3D
}

\author{
$M^{a}$ Paz Prendes Espinosa \\ Universidad de Murcia \\ pazprend@um.es
}

"La mayor aventura es la que nos espera.
Hoy y mañana aún no se han dicho".
El Hobbit
J.R.R. Tolkien

\section{HABLANDO DE TECNOLOGÍA EDUCATIVA}

Hablar de "Tecnología Educativa" supone referirse a un ámbito de conocimiento desde el cual se genera un espacio para la investigación, un espacio para la docencia y un espacio para la innovación educativa apoyada en tecnologías. Esta visión tridimensional de nuestra disciplina es la que probablemente influye en su cada vez mayor relevancia en el contexto de la Pedagogía.

Los orígenes de la Tecnología Educativa son bien conocidos y nos remontan a la psicología y su afán por encontrar puentes conceptuales entre el aprendizaje y los medios de enseñanza. En mis tiempos de estudiante de Pedagogía -allá en la década de los 80- me llamaban poderosamente la atención los vídeos de Skinner y sus máquinas de enseñar (uno de ellos puede verse subtitulado en español en este enlace). Pasadas las etapas históricas focalizadas en los medios audiovisuales y la enseñanza programada en los años 50 y 60 , el auge de los medios de comunicación de masas en los 70 y 80, o la informática en los años 90, los albores del nuevo siglo llegaron con una revolucionaria Tecnología Educativa centrada en los cambios asociados a Internet, Web, Redes Sociales...

Este auge de la Tecnología Educativa ha ido generando un gran interés que en cierta manera nos empuja a reflexionar sobre su sentido y a redibujar sus límites. Porque la Tecnología Educativa no es usar internet o adquirir competencias digitales, sino que va mucho más allá.

Si analizamos la Tecnología Educativa desde su dimensión epistemológica, observamos cómo han ido incorporándose disciplinas nuevas a su construcción teórica. En sus orígenes, la Tecnología Educativa se apoyaba principalmente en las Teorías de la Educación, la Psicología del Aprendizaje, las Teorías de la Comunicación y la Teoría de Sistemas, pero estos pilares de la Tecnología Educativa han ido progresivamente abriendo el camino a otras disciplinas que han marcado la evolución de la propia Tecnología Educativa.

Así hemos visto, por ejemplo, cómo la teoría de sistemas ha ido perdiendo peso desde los años 80 . Hemos podido observar la creciente influencia de la Sociología conforme se ha ido conectando la Tecnología Educativa a los aspectos sociales, especialmente desde su vinculación al movimiento CTS (Ciencia, Tecnología y Sociedad) en los 90. O la influencia, percibida en todas las ciencias de la educación, de la Escuela de Frankfurt y el movimiento socio-crítico. Hemos visto también cómo las teorías de la comunicación se han ido uniendo a teorías psicológicas, conformando modelos como el conectivismo o el sharismo que son de relevancia en la fundamentación teórica actual de la Tecnología Educativa. Por su parte la cibernética, que 
parecía que había perdido en cierta medida su fuerza, en los últimos años hemos ido viendo cómo ha recobrado un renovado impulso y cómo los desarrollos de la inteligencia artificial (IA) le han hecho recobrar protagonismo. Y es importante reconocer la importancia de la telemática en los últimos años del siglo XX y los más recientes del siglo XXI. A todas estas disciplinas se añaden otras, como pueden ser en la actualidad la Lingüística o la Teoría del Género.

La tradición de la Pedagogía en España históricamente ha situado a la Tecnología Educativa en el ámbito del área de la Didáctica y la Organización Escolar, quedando por tanto desligada de la Psicología que la vio nacer. En la Didáctica hemos encontrado los tecnólogos educativos un sustento teórico para explicar la enseñanza como proceso de comunicación y para analizar cómo los medios influyen en ese proceso -reconfigurando tanto las decisiones sobre enseñanza del docente como los aprendizajes de los discentes-. Pero conforme las tecnologías han ido provocando un mayor impacto educativo y social, la Tecnología Educativa ha ido adquiriendo una mayor relevancia en el mundo real de la educación, una relevancia no obstante no siempre reconocida en los planes de estudios de formación inicial del profesorado o en los planes de estudios de los futuros expertos en educación (pedagogos y educadores sociales).

Y todas estas bases teóricas de la Tecnología Educativa nos ayudarán a construir nuestros espacios de investigación, de docencia y de innovación que vamos a comentar a continuación, una visión tridimensional de la Tecnología Educativa que quizás pueda servir como sustento para comprender mejor su pasado y su presente, así como para reconstruirla mirando hacia su prometedor futuro.

\section{LA TECNOLOGÍA EDUCATIVA COMO ESPACIO PARA LA DOCENCIA}

Desde una perspectiva de análisis disciplinar, observamos que la Tecnología Educativa no siempre aparece en los planes de estudio como tal, encontrando denominaciones diversas como "Innovaciones tecnológicas y educación", "Medios audiovisuales y TIC", "Recursos y TIC", "TIC para la formación", "TIC en Educación Social",... (véase la presentación realizada en Valencia en 2013: https://goo.gl/4cEYsX). En este sentido es importante recordar la advertencia de Martínez (2016, p. 21): "Las TIC han logrado situarse como una especialidad en sí mismas creando un distanciamiento, cuando no un olvido de la TE [Tecnología Educativa], con lo que ello supone de empobrecimiento conceptual y de justificación pedagógica de aquéllas dentro de la acción didáctica". Los mecanismos por los cuales se diseñan los planes de estudios tristemente no siempre responden a criterios ni normativos, ni científicos, ni académicos. Más bien al contrario, en estas situaciones se ponen de manifiesto intereses personales y conflictos internos que los académicos deberíamos ser capaces de dejar al margen por el bien de la formación de nuestro alumnado.

Aunque somos conscientes de las dificultades de elaborar un plan de estudios, es necesario reconocer la importancia de los informes internacionales sobre la formación en Tecnología Educativa de los formadores y especialistas en educación (UNESCO, 2004; Unión Europea, 2005; Comisión Europea, 2006; Gómez, 2016). Y tal y como recogen Fernández, Fernández y Cebreiro (2016, p. 216), "son muchos los estudios que han demostrado la carencia formativa del profesorado en TIC", poniéndose de manifiesto que por lo general sí están formados para el uso técnico de las herramientas tecnológicas, pero no están formados en la dimensión didáctica y pedagógica necesaria para integrar las TIC en las aulas. Estos datos parecen corroborarse en la investigación llevada a cabo con profesorado no universitario (García-Valcárcel y TejedorTejedor, 2005) en la cual describen que el profesorado hace un uso "razonable y en parámetros de normalidad" de las TIC en su contexto personal, pero por el contrario "el uso que los profesores hacen de las TIC en el aula puede considerarse muy bajo; los porcentajes, salvo en el caso de los medios audiovisuales, con mayor implantación, son francamente bajos" (p. 130). 
Esta formación en competencias técnicas aparece en consonancia con el espejismo que ejercen las TIC, provocando que la Tecnología Educativa se desdibuje y "poniendo el foco de atención en la parte en vez de en el todo" (Sancho, Bosco, Alonso y Sánchez, 2015, p. 18). En palabras de los autores,

"Considerar que la TE [Tecnología Educativa] es el último artefacto o aplicación que mejora el procesamiento de la información tiene dos consecuencias inmediatas. La primera y más preocupante es que nos lleva a olvidar o considerar el potente dispositivo que son hoy las instituciones educativas. [...] La segunda nos lleva a alimentar mitos, en forma de relatos fabulosos y supuestos epistemológicos carentes de evidencia empírica y contraste con la realidad" (pp. 18-19).

Por tanto, es una necesidad primordial que en las revisiones de los planes de estudios en Educación sepamos darle a la Tecnología Educativa el valor que sería deseable en relación a las demandas competenciales del mercado laboral, en relación a las demandas de la sociedad actual del aprendizaje permanente y en relación, por último, a la propia consideración de la disciplina no desde un carácter instrumental, sino desde su propia identidad epistemológica. Es probable que en estos procesos de diseño curricular nos encontremos con quienes destaquen "por su clarividencia y altura de miras", pero también es probable que nos encontremos con aquellos otros que destacarán "por su codicia y miopía" (Trillo, 2008, p. 23).

En esta revisión sería importante no solamente el hecho de intentar recuperar la denominación propia de la disciplina en los planes de estudio, sino reflexionar sobre los contenidos y las actividades que trabajamos con nuestros alumnos de las titulaciones de Educación. Las tareas de reflexión y análisis de modelos y teorías han ido perdiendo espacio en favor de tareas centradas más en cuestiones técnicas y producción de "artefactos". Y con ello la parte más tecnológica ha ido absorbiendo el espacio del contenido didáctico y pedagógico de nuestra disciplina.

Más allá de la formación específica en las titulaciones de Educación, es fácil reconocer también su presencia en otros espacios formativos universitarios. Las universidades han visto en los últimos años cómo las tecnologías digitales han influido en los procesos formativos, en la investigación y también en la gestión y administración de la institución. Y es por esta omnipresencia de las tecnologías que en paralelo ha surgido la necesidad de incrementar la formación tecnológica de todo el personal universitario, tanto PDI como PAS, lo que dibuja una foto fija en la que todos los colectivos universitarios reconocen de modo implícito la necesidad de aprender a usar tecnologías. Este uso de las tecnologías es, como decimos, variado y diverso, pues además de su utilidad en procedimientos de gestión, de docencia o de investigación, es fundamental descubrir y reconocer su relevancia en cuanto a la formación permanente, el aprendizaje autorregulado, su uso como herramientas de comunicación y divulgación científica, herramientas para construir redes personales y profesionales y, en general, para cultivar nuestro crecimiento profesional a lo largo de la vida.

\section{LA TECNOLOGÍA EDUCATIVA COMO ESPACIO PARA LA INNOVACIÓN}

En segundo lugar, el análisis de la innovación educativa apoyada en tecnologías nos dibuja un espacio de aplicación práctica de la Tecnología Educativa que no tiene fronteras, un contexto en el cual la Tecnología Educativa sustenta su acción práctica y que en los últimos años aparece muy ligada a los desarrollos de la telemática, la informática, las tecnologías digitales y las redes. La Tecnología Educativa ha de ser vista como una disciplina integradora de conocimientos que sirven para sustentar procesos reales de innovación con tecnologías en cualquier nivel de la enseñanza y en relación a contextos formales, no formales e informales.

En relación con este aspecto de la innovación apoyada en tecnologías, como condición previa y necesaria es obvio que el primer requerimiento es disponer de los medios tecnológicos, 
condición que en general en las instituciones de enseñanza en Europa se cumple y especialmente en las universidades. En la "Agenda Digital para Europa" de la Comisión Europea (2014, p. 3) se explica que "la economía digital crece siete veces más deprisa que el resto de la economía" y se prevé que en 2020 tendremos "16 millones más de puestos de trabajo que requieran competencias en tecnologías de la información y la comunicación". En este mismo informe se recoge el dato de que ya en 2014 la cobertura básica de banda ancha era del 100\% en Europa. En cuanto al uso de Internet (Figura 1), el Instituto Nacional de Estadística muestra datos de casi el 99\% de la población en las horquillas de edad que van desde los 16 hasta los 34 años, siendo a partir de esa edad superiores al 90\% y algo más reducidas a partir de los 55 años (INE, 2016).

Figura 1. Usuarios de Internet en España en 2016 (INE, 2016)

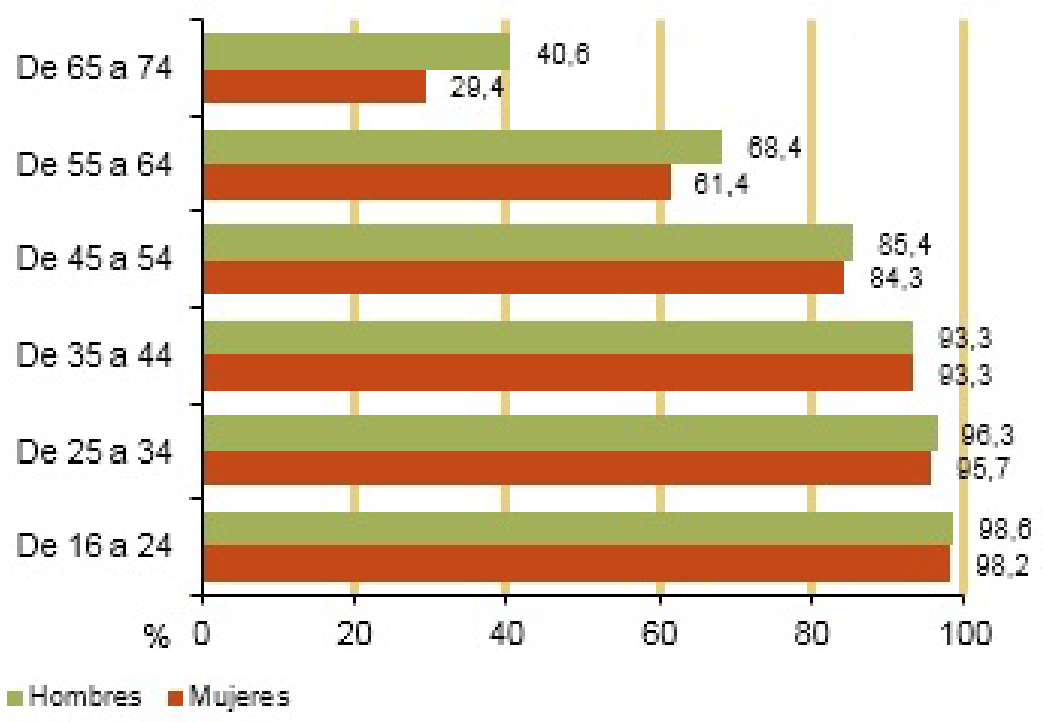

Según los datos de Eurydice (2011) a partir de un estudio con 35 países, la mayor parte de los centros de enseñanza en Europa cuentan con tecnologías y no hay grandes diferencias en cuanto a equipamiento. En este informe se argumenta la importancia de la innovación en las instituciones de enseñanza y, de forma más específica, las innovaciones orientadas a la mejora de la enseñanza y el aprendizaje mediante la utilización de nuevas tecnologías. Situando nuestro punto de mira en las universidades españolas, los datos de CRUE-TIC (2017) vienen a describir esta misma realidad, el equipamiento y las infraestructuras son una condición necesaria pero no son un problema.

Una vez que los datos muestran que contamos con la infraestructura tecnológica necesaria para promover cambios y mejoras, será importante concretar hacia dónde queremos que las tecnologías nos lleven. Las tecnologías no han de ser, en ningún caso, un fin en sí mismas, sino que han de ser las herramientas para alcanzar los objetivos estratégicos de las instituciones y de las políticas educativas locales, nacionales e internacionales, a sabiendas de que su bondad o maldad vendrá condicionada por el uso que hagamos de ellas. Y uno de estos buenos usos de las tecnologías viene determinado por el loable afán de innovar en educación en todos sus niveles y espacios formales, no formales e informales.

Entendemos la innovación, por una parte, como un proceso de cambio asociado de forma incuestionable a la mejora. En palabras de Zabalza (2004, p. 120), la innovación es "apertura, actualización, mejora". En este mismo sentido Casas y Stojanovic (2013, p. 62) abundan en la 
idea de que "la innovación es un cambio pensado para mejorar, reformar y lograr resultados institucionales más efectivos que los actuales", entendiendo así la innovación como un proceso social de la organización y no como un elemento individual.

En esta línea de pensamiento, podemos considerar la innovación desde una óptica estructural, es decir, la innovación como objetivo estratégico que condiciona y determina las políticas y los marcos para los procesos de toma de decisión. O bien de forma complementaria podemos analizar la innovación desde una óptica micro, la innovación en este caso como una actitud y una iniciativa de las personas que integran esas organizaciones. Así pues, es probable que todos conozcamos organizaciones que diseñan estrategias de innovación institucional y, en otros casos, profesores que son muy innovadores a pesar de que sus instituciones no les ayudan ni lo promueven. En relación con este último caso, podemos colegir con Trillo (2008, p. 39) que "del mismo modo que un árbol no hace un bosque, la innovación que descansa sobre profesores individuales no caracteriza todavía al centro como tal".

En el ámbito específico de la enseñanza, la innovación docente es entendida como "la capacidad para crear y aplicar nuevos conocimientos, perspectivas, metodologías y recursos en las diferentes dimensiones de la actividad docente, orientadas a la mejora de la calidad del proceso de enseñanza-aprendizaje" (Pagés y cols., 2016, p. 35). En el estudio llevado a cabo por estas autoras con una muestra representativa del profesorado universitario en España, se concluye que las ramas de conocimiento donde el profesorado más valora la innovación docente son Ciencias de la Salud y en segundo lugar Ciencias Sociales y Jurídicas. Los que menos valoraron la innovación docente fueron los de Ciencias, Ingeniería y Arquitectura.

Estos procesos de innovación educativa apoyados en tecnologías podrían ser uno de los ejes de cambio de las instituciones de enseñanza en el futuro a corto, medio y largo plazo. "Los sistemas educativos de todo el mundo se enfrentan actualmente al desafío de utilizar las nuevas tecnologías de la información y la comunicación" (UNESCO, 2004, p. 13). Y estos cambios institucionales no deben ser pensados desde una óptica individual, sino que la innovación educativa afecta a estrategias, procedimientos, recursos, metodologías, organización,... todos los elementos que conforman el sistema complejo de las organizaciones e instituciones educativas en el complejo cosmos de sus sistemas sociales.

Por otra parte, este enfoque organizativo de las innovaciones educativas puede responder a un modelo cerrado (aquellos procesos que se limitan a mejorar la propia organización) o a modelos abiertos (que buscan el impacto en el exterior y más allá de la organización en la cual surgen y se desarrollan). Ejemplos de este segundo caso serían las comunidades Open Course Ware en el ámbito de la enseñanza superior.

Figura 2. Innovación apoyada en TIC

\begin{tabular}{|c|c|c|}
\hline Formación & Investigación & Gestión \\
\hline $\begin{array}{l}\text { - Recursos digitales } \\
\text { abiertos } \\
\text { - PDI } \\
\text { - Enseñanza flexible } \\
\text { - E-learning } \\
\text { - B-learning } \\
\text { - M-learning } \\
\text { - Comunidades de } \\
\text { aprendizaje } \\
\text { - Redes docentes }\end{array}$ & $\begin{array}{l}\text { - Gestión de la } \\
\text { información } \\
\text { - Análisis de datos } \\
\text { - Redes de } \\
\text { investigación } \\
\text { - Comunidades } \\
\text { abiertas } \\
\text { - Difusión en línea } \\
\text { - Bases de datos } \\
\text { - Revistas digitales }\end{array}$ & $\begin{array}{l}\text { - Administración } \\
\text { electrónica } \\
\text { - Almacenamiento } \\
\text { de datos } \\
\text { - Gestión de la } \\
\text { información } \\
\text { - Repositorios } \\
\text { documentales } \\
\text { - Certificación y } \\
\text { autentificación } \\
\text { documental }\end{array}$ \\
\hline \multicolumn{3}{|c|}{ TIC para la INNOVACIÓN } \\
\hline
\end{tabular}


En los últimos años, en el nivel de la enseñanza superior hemos observado cómo las tecnologías han supuesto un gran avance en relación con la administración electrónica y cómo se han convertido en herramienta imprescindible de investigación. En otros niveles de enseñanza cada vez con mayor frecuencia encontramos profesores innovadores que han convertido las tecnologías en medios propios de su realidad diaria en las aulas. Y en el sistema educativo en general, todos los documentos normativos y legislativos reconocen oficialmente la necesidad de trabajar con Tecnologías de la Información y la Comunicación. En todos esos procesos, la Tecnología Educativa tiene mucho que decir.

\section{LA TECNOLOGÍA EDUCATIVA COMO ESPACIO PARA LA INVESTIGACIÓN}

Las líneas de investigación de la Tecnología Educativa son, como en cualquier otra disciplina, cambiantes y es por ello que hemos ido dejando atrás la investigación sobre la enseñanza programada, la teoría del esfuerzo invertido, las comparaciones de medios, el valor didáctico de los diaporamas o el uso del vídeo en el aula, por poner algunos ejemplos, y hemos ido evolucionando hacia Internet, la enseñanza virtual o la innovación con nuevas tecnologías. En un estudio que realizamos en 1998 sobre las líneas de investigación en Tecnología Educativa en España, encontrábamos como focos de interés los siguientes: cuestiones conceptuales de la Tecnología Educativa; análisis de medios; diseño y evaluación de materiales; la formación del profesorado para el uso de tecnologías; y por último, experiencias de integración de tecnologías. Para entender los cambios y evolución en estos últimos años, hemos recogido las conclusiones de diversos estudios bibliográficos y meta-análisis (Hsu, Hung y Ching, 2013; Baydas et al., 2015; Cabero, 2016; Valverde, 2016; Escalona, Gómez y Escalona, 2017).

Hsu, Hung y Ching (2013) analizan los resúmenes de 2.997 artículos publicados entre el año 2000 y el 2010 en seis revistas internacionales del SSCI (todas ellas en inglés). De sus conclusiones nos parece especialmente relevante destacar que los tres tópicos de investigación más frecuentes encontrados por los autores son: 1) la integración de tecnologías, 2) las actitudes y aceptación de tecnologías y 3 ) los entornos de aprendizaje. No obstante, su estudio indica que probablemente el tópico relacionado con las actitudes no crezca en los próximos años, mientras que por el contrario la investigación sobre aspectos pedagógicos del uso de tecnologías y la efectividad de las estrategias de aprendizaje sí podrían incrementar su interés en la investigación. $Y$ dentro de estos temas, se destaca el volumen de investigaciones sobre estrategias de enseñanza basadas en la interacción y el trabajo colaborativo.

En el análisis sobre investigaciones en Tecnología Educativa realizado por Baydas, Kucuk, Yilmaz, Aydemir y Goktas (2015) también encontramos interesantes datos. Los autores estudian las dos revistas internacionales que ellos consideran más relevantes (igualmente en inglés) y realizan un análisis de contenido de todos los artículos publicados en el periodo 2002-2014, un total de 1.255 artículos. Llegan a la conclusión de que los temas más frecuentes son los enfoques y teorías sobre el aprendizaje, por una parte, y en segundo lugar, los entornos de aprendizaje (coincidiendo con Hsu, Hung y Ching en este último tópico). Esta conclusión nos lleva irremediablemente a pensar que: primero, la psicología está de nuevo asumiendo un gran papel protagonista en la evolución de la Tecnología Educativa en estos últimos años; y segundo, la mayoría de los estudios sobre PLE tienen un marcado enfoque técnico y tecnológico. Estos dos factores sumados nos ponen sobre la pista de que quizás estamos perdiendo esa visión más pedagógica y social que la Tecnología Educativa sí parecía tener en los años 90. Con porcentajes más bajos que las dos anteriores, pero con datos también importantes, aparece el tema del elearning.

En su meta-análisis, Baydas y sus colaboradores también señalan que los instrumentos de recogida de datos más utilizados son los cuestionarios, el análisis documental y las entrevistas. Y la mayor parte de los estudios son de corte cuantitativo, tal y como se observa en la Figura 3. 
Figura 3. Métodos de investigación en Tecnología Educativa según el análisis de Baydas et al. (2015, p. 720).

En el trabajo de Cabero (2016), realizado a partir de la recopilación de datos de investigaciones previas basadas en la técnica del meta-análisis, se remarca que se ha producido un incremento volumétrico de investigaciones que, sin embargo, no ha venido aparejado de resultados significativos en cuanto a la aplicación del conocimiento en la integración de TIC en educación: "los resultados no han sido siempre muy significativos para aumentar el conocimiento científico respecto a la integración de las TIC en la práctica educativa" (p. 24). En este mismo sentido abunda Gros al afirmar que "después de treinta años de investigación, todavía hay una ingenua suposición de que la tecnología por sí misma mejora el aprendizaje" (2012, p. 2).

Valverde (2016) coincide con esta visión crítica de la utilidad real de la investigación en nuestro campo y también señala que "los miles de estudios en TE [Tecnología Educativa] y metaanálisis de estas investigaciones han demostrado que la TE no ha alcanzado aún su supuesto potencial y, en los últimos años, el escepticismo sobre su eficacia se ha incrementado", a lo que añade la idea de que "los investigadores en educación parecemos ser incapaces de aprender de nuestra historia pasada y del insignificante impacto en la práctica educativa de nuestros estudios" (p. 62). Como conclusión, el autor señala que es importante revisar nuestro modo de investigar en Tecnología Educativa y que deberíamos estudiar problemas complejos, analizar contextos reales, colaborar con los profesores, integrar factores de diseño instruccional junto con las tecnologías, promover estudios rigurosos, reflexionar sobre los resultados, refinar entornos innovadores para el aprendizaje y, por último, ser capaces de concretar nuevos principios educativos.

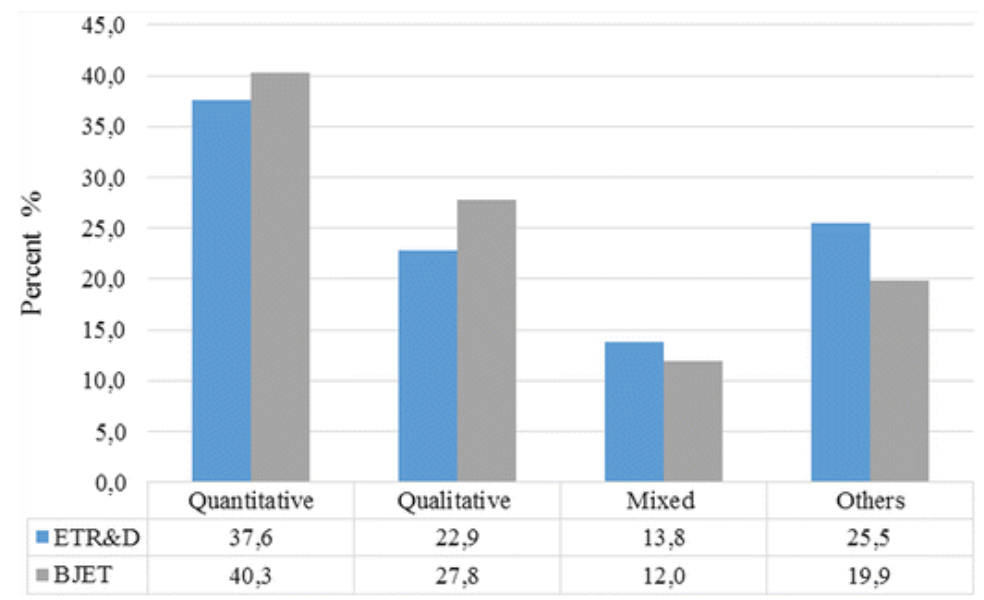

Cabero indica que estas investigaciones nos han enseñado: 1) que debemos superar el modelo comparativo de medios; 2) que por lo general las investigaciones se desarrollan con escasa fundamentación teórica; 3) que es importante realizar investigaciones con metodologías mixtas y técnicas diversas; 4) que existe una tendencia hacia los estudios sistémicos; y 5) que se debería reflexionar sobre la ampliación de los informantes (Cabero, 2016, pp. 26-29). Entre sus reflexiones sobre la evolución de la investigación en Tecnología Educativa, el autor afirma:

"En su evolución no podemos olvidarnos que se han pasado por diferentes estadios, que han ido desde los estudios comparativos, el análisis de los atributos estructurales de los medios, el estudio de los atributos simbólicos y las interacciones que se establecen con los estudiantes, la significación adquirida por las actitudes hacia los medios, la importancia de los contextos para la concreción de las tecnologías, el estudio de las estrategias de aplicación de los medios, los 
criterios que los profesores aplican para la incorporación de las TIC y la incorporación de las TIC desde la perspectiva sociocrítica" (Cabero, 2016, p. 25).

Todos estos estudios nos llevan a concluir que sí se ha producido una evolución en las líneas y temas de investigación en la Tecnología Educativa en los últimos veinte años. Por una parte, parece que ha dejado de tener interés el estudio teórico sobre la propia Tecnología educativa y el análisis de los medios audiovisuales. Y han aparecido con fuerza los tópicos de investigación relacionados con los ambientes y entornos de aprendizaje virtuales. Valverde lo sintetiza afirmando que:

\begin{abstract}
"actualmente la investigación en TE se orienta especialmente hacia el diseño de entornos que fomentan el aprendizaje con la participación-reflexión de todos los agentes implicados en contextos auténticos y con la finalidad de comprender los fenómenos y aportar soluciones a problemas reales. Por otra parte, la ética establece las bases para la práctica, constituye un enfoque desde el que desarrollar la TE e implica un compromiso individual, social y profesional" (Valverde, 2015, p. 12)
\end{abstract}

Figura 4. Evolución de la investigación en Tecnología Educativa en los últimos veinte años.

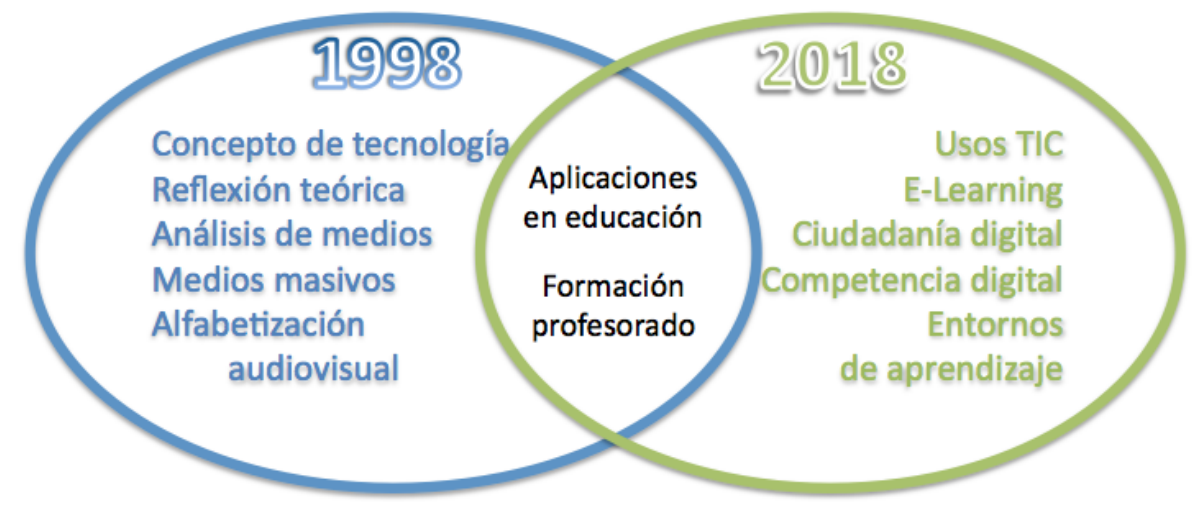

\title{
5. INVESTIGAR Y DIFUNDIR LA INVESTIGACIÓN
}

En este marco de interrelaciones se va construyendo el conocimiento propio y la investigación en Tecnología Educativa, un marco que es muy importante que los investigadores noveles conozcan, pues no es posible entender el presente sin conocer el pasado. $Y$ este conocimiento nos evitará repetir errores o pensar que hemos descubierto la pólvora cuando resulta que otros la habían descubierto antes.

La Tecnología Educativa tiene un futuro prometedor e igual de apasionante que su historia pasada, pero es importante que aquellos con responsabilidad en la definición de la disciplina y en la construcción de su espacio propio asuman que el simple uso de medios tecnológicos no supone hablar de Tecnología Educativa. Es importante que fijemos nuestra atención en los modelos teóricos que sustentan la acción pedagógica apoyada en tecnologías y la acción didáctica configurada como proceso mediado. $Y$ ese sustento teórico nos capacitará para la reconstrucción del conocimiento y para el avance y la innovación en las prácticas reales del mundo educativo. Este modelo 3D (la Tecnología Educativa como espacio para la docencia, espacio para la investigación y espacio para la innovación) lo hemos intentado recoger de forma esquemática en la Figura 5. 
Figura 5. Las tres dimensiones de la Tecnología Educativa: docencia, investigación e innovación.

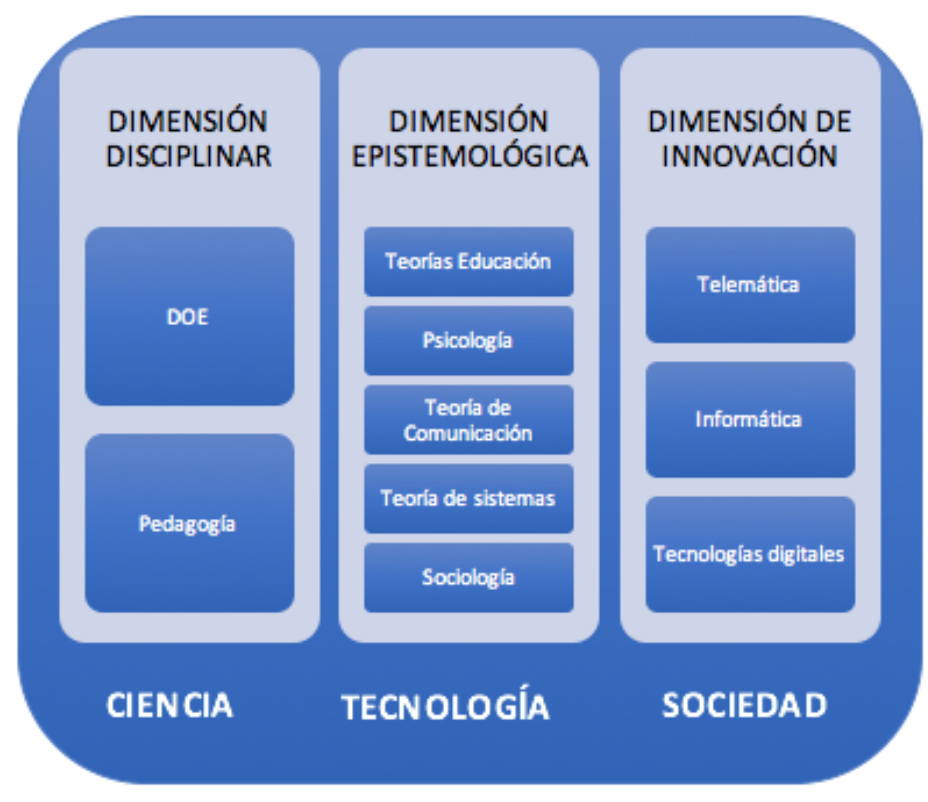

Pero además de investigar, hay que contribuir a la difusión de la investigación y a ello dedicamos nuestro esfuerzo en RIITE, revista que cada semestre recoge resultados de investigaciones en el ámbito de la Tecnología Educativa y que contribuye al esfuerzo de los investigadores noveles, quienes a menudo tan difícil encuentran el acceso al mundo de las publicaciones.

Y llegamos ya al número 4 de la revista, en el cual se recogen las siguientes contribuciones:

- Una entrevista que Marimar Román realiza a Ricardo Román, entre quienes aseguramos que no hay parentesco familiar. Agradecemos mucho a Ricardo que haya aceptado participar en este número de la Revista y les aseguramos que es muy interesante leer sus reflexiones sobre la educación en general y sobre las tecnologías y su impacto educativo en particular.

- Christian Rodríguez y Esther del Moral publican un estudio sobre videojuegos multijugador realizado con una metodología mixta y centrándose en el papel clave del entrenador experto.

- El artículo sobre uso de tecnologías con niños con TEA ha sido presentado por Sanromá-Giménez, Lázaro-Cantabrana y Gisbert-Cervera, un trabajo en el cual presentan un instrumento para evaluar el uso de aplicaciones móviles.

- La investigación de Padilla, Gámiz y Romero se centra en el estudio de la Competencia Digital Docente y presentan una propuesta de entrevista para el profesorado universitario.

- J.M. Falcó y J.L. Huertas han realizado una evaluación de su experiencia de ludificación con estudiantes universitarios.

- El trabajo de Francisco L. Saorín e Isabel Gutiérrez sobre la identidad digital del alumnado universitario, un estudio realizado con estudiantes de Educación en la Universidad de Murcia.

- La reseña de este número corre a cargo de Victoria Marín, quien resume y valora el libro sobre "Entornos Personales de Aprendizaje: una visión actual de cómo aprender con tecnologías" publicado por Octaedro en 2017. 
Agradecemos a todos los autores su confianza en RIITE para publicar sus investigaciones. Y también agradecemos el esfuerzo de difusión de los trabajos de toda la Comunidad RIITE a través de nuestras redes sociales.

\section{REFERENCIAS BIBLIOGRÁFICAS}

Baydas, O., Kucuk, S., Yilmaz, R. M., Aydemir, M., y Goktas, Y. (2015). Educational technology research trends from 2002 to 2014. Scientometrics, 105(1), 709-725. DOI: 10.1007/s11192015-1693-4

Cabero, J. (2016). ¿Qué debemos aprender de las pasadas investigaciones en Tecnología Educativa? RIITE, Revista Interuniversitaria de Investigación en Tecnología Educativa, 0, pp. 23-33. DOI: 10.6018/riite/2016/256741

Casas, M. y Stojanovic, L. (2013). Innovación en la universidad iberoamericana. Revista de Universidad y Sociedad del Conocimiento (RUSC), 10(1), 61-74.

Comisión Europea (2006). Competencias clave para el aprendizaje permanente. Recomendación 2006/962/CE del Parlamento Europeo y del Consejo, de 18 de diciembre de 2006, sobre las competencias clave para el aprendizaje permanente [Diario Oficial L 394 de 30.12.2006]

Comisión Europea (2014). Comprender las políticas de la Unión Europea: Agenda Digital para Europa. Recuperado de http://europa.eu/pol/index es.htm

Coll, C. (2010). Enseñar y aprender en el mundo actual: desafíos y encrucijadas. Pensamiento Iberoamericano, 7, 47-66. Recuperado de http://www.educacionysociedad.org/images/img noticias/docu4e92a454ee178 101020114 $\underline{52 a m . p d f}$

Eurydice (2011). Key Data on Learning and Innovation through ICT at School in Europe 2011. Recuperado de http://eacea.ec.europa.eu/education/eurydice

Fernández de la Iglesia, C., Fernández Morante, C. y Cebreiro López, B. (2016). Competencias en TIC del profesorado en Galicia: Variables que inciden en las necesidades formativas. Innovación Educativa, 26, 215-231. Recuperado de http://www.usc.es/revistas/index.php/ie/article/viewFile/3256/3927

García-Valcárcel, A. y Tejedor-Tejedor, J. (2005). Condicionantes (actitudes, conocimientos, usos, intereses, necesidades formativas) a tener en cuenta en la formación del profesorado no universitario en TIC. Enseñanza, (23), 115-142. Recuperado de http://revistas.usal.es/index.php/0212-5374/article/view/4216

Gómez, J. (2016). UNIVERSITIC 2016. Análisis de las TIC en las Universidades Españolas. Madrid: CRUE Universidades Españolas. Recuperado de http://tic.crue.org/wpcontent/uploads/2017/04/UNIVERSITIC-2016-con-portadas.pdf

Gros, B. (2012). Retos y tendencias sobre el futuro de la investigación acerca del aprendizaje con tecnologías digitales. RED. Revista de Educación a Distancia, 32. Recuperado de: http://www.um.es/ead/red/32/gros.pdf

Hsu, Y.C., Hung, J.L. y Ching, Y.H. (2013). Trends of educational technology research: more than a decade of international research in six SSCl-indexed refereed journals. Educational Technology Research and Development, 61(4), 685-705. DOI: 10.1007/s11423-013-9290-9

INE, Instituto Nacional de Estadística (2016). Encuesta sobre Equipamiento y Uso de Tecnologías de la Información y Comunicación en los Hogares. Recuperado de http://bit.ly/1PYwho6

Martínez Sánchez, F. (2016). Sentado en el andén. RIITE. Revista Interuniversitaria de Investigación en Tecnología Educativa, 0, 17-22. DOI: 10.6018/riite/2016/258131 
Pagés, T., Hernández, C., Abadía, A.R., Bueno, C., Ubieto-Artur, I., Márquez, D., Sabaté, S. y Jorba, H. (2016). La innovación como competencia docente en la universidad: innovación orientada a la mejora de aprendizaje. Revista de Psicología, Ciencies de l'Edució i de l'Esport, 34(1), 33-43.

Sancho Gil, J., Bosco Paniagua, A., Alonso Cano, C. y Sánchez Valero, J.A. (2015). Formación del profesorado en Tecnología Educativa: de cómo las realidades generan los mitos. RELATEC, Revista Latinoamericana de Tecnología Educativa, 14(1), 17-30. Recuperado de http://relatec.unex.es

Trillo, F. (2008). El reto de las titulaciones de educación. Otra crónica a 7 de marzo del 2008. Educación XXI, 11, 19-41. Recuperado de http://revistas.uned.es/index.php/educacionXX1/article/view/308/264

UNESCO (2004). Las Tecnologías de la Información y la Comunicación en la formación docente. Guía de Planificación. Recuperado de http://unesdoc.unesco.org/images/0012/001295/129533s.pdf

Unión Europea (2005). Recomendación del Parlamento Europeo y del Consejo sobre las competencias clave para el aprendizaje permanente. Bruselas. Documento en línea en http://www.crue.org/export/sites/Crue/procbolonia/documentos/antecedentes/9. Competenci as clave para aprendizaje permanente.pdf

Valverde-Berrocoso, J. (2015). Presentación. La formación universitaria en Tecnología Educativa: enfoques, perspectivas e innovación. RELATEC Revista Latinoamericana de Tecnología Educativa, 14(1), 11-16. Recuperado de http://relatec.unex.es

Valverde-Berrocoso, J. (2016). La investigación en Tecnología Educativa y las nuevas ecologías del aprendizaje: Design-Based Research (DBR) como enfoque metodológico. RIITE, Revista Interuniversitaria de Investigación en Tecnología Educativa, 0, 60-73. Doi: http://dx.doi.org/10.6018/riite/2016/257931

Zabalza, M.A. (2004). Innovación en la enseñanza universitaria. Contextos Educativos, 6-7, 113136.

\section{INFORMACIÓN SOBRE LA AUTORA}

\section{Ma Paz Prendes Espinosa}

Universidad de Murcia

Doctora en Ciencias de la Educación. Catedrática de Didáctica y Organización Escolar y Directora del Grupo de Investigación en Tecnología Educativa de la Universidad de Murcia. Coordinadora de los programas interuniversitarios de Máster y Doctorado en Tecnología Educativa en la Universidad de Murcia. Secretaria de la Asociación Edutec para el Desarrollo de la Tecnología Educativa. Directora de RIITE, Revista Interuniversitaria de Investigación en Tecnología Educativa. Para más información: http://www.pazprendes.es/, http://www.um.es/gite/ y @pazprendes

\section{(1) (9)}

Los textos publicados en esta revista están sujetos a una licencia de Reconocimiento 4.0 España de Creative Commons. Puede copiarlos, distribuirlos, comunicarlos públicamente y hacer obras derivadas siempre que reconozca los créditos de las obras (autoría, nombre de la revista, institución editora) de la manera especificada por los autores o por la revista. La licencia completa se puede consultar en:Licencia Creative Commons Atribución-NoComercial-Compartir por igual 4.0 Internacional. 